\title{
SOLAR ABUNDANCES OF C, N, AND O
}

\author{
N. GREVESSE \\ Institut d'Astrophysique, Université de Liège, \\ avenue de Cointe, 5 B-4000 Liège, Belgium \\ and \\ A. J. SAUVAL and R. BLOMME \\ Observatoire Royal de Belgique/Koninklijke Sterrenwacht van België, \\ B-1180 Brussels, Belgium
}

\begin{abstract}
We briefly review the many indicators, atoms as well as molecules, of the photospheric abundances of $\mathrm{C}, \mathrm{N}$ and $\mathrm{O}$ and present preliminary updated values of these abundances.
\end{abstract}

Key words: C, N, O - infrared: stars - stars: abundances - Sun: abundances

\section{Introduction}

We briefly recall the main reasons why accurate values of the solar abundances of $\mathrm{C}, \mathrm{N}$ and $\mathrm{O}$ are so important.

They contribute about $70 \%$ to the metallicity $\mathrm{Z}$; their detailed contribution to this metallicity is of crucial importance, as has been shown by the new opacity calculations for stellar interiors like OPAL (Iglesias and Rogers, 1991) and the OPACITY PROJECT (Seaton et al., 1992) and for stellar envelopes (Kurucz, 1991).

The solar photosphere is the only reliable source for the abundances of these elements. It is well known that they have partially escaped from meteorites. Furthermore, although their abundances can be derived from the coronal spectrum and from solar wind and solar energetic particles data, the fractionation process between the outer solar layers and the photosphere makes accurate comparisons difficult (see e.g. Anders and Grevesse, 1989).

It is also well known that the $\mathrm{C} / \mathrm{O}$ ratio is a crucial parameter for the physicochemistry during the early phases of the evolution of the solar system.

\section{Indicators of the Solar $\mathrm{C}, \mathrm{N}$ and $\mathrm{O}$ Abundances}

With the availability of the ATMOS infrared solar spectra obtained from space (Farmer and Norton, 1989) and covering the region from 2 to $16 \mu \mathrm{m}$, we have been able to use all the best indicators of the abundances of these elements, i.e., atomic as well as molecular lines. The best indicators should have reliable atomic data (gf-values) and/or molecular data (transition probabilities, dissociation energies) as well as solar data (equivalent widths). We therefore disregarded some transitions in the visible spectrum which are too difficult to measure with accuracy in the solar spectrum because of the increasing blending problem as one goes to shorter wavelengths. The indicators we retained are the following: $\mathrm{C} \mathrm{I}$ and [C I], N I, O I and [O I], CH (A-X), CH vibration-rotation, $\mathrm{C}_{2}$ (Swan, Phillips and Ballik-Ramsay), $\mathrm{NH}$ vibration-rotation and pure rotation, $\mathrm{OH}$ vibration-rotation and pure rotation, $\mathrm{CN}$ red system, $\mathrm{CO}$ vibration-rotation $(\Delta v=1$ and 2$)$. Most of these indicators are in the infrared. 


\section{This Work}

In several recent papers (Sauval et al., 1984; Grevesse et al., 1984; Grevesse et al., 1990, 1991; Geller et al., 1991; Grevesse and Sauval, 1991) we have analyzed these indicators and have shown how remarkably well they lead to the same results. It also became clear from these, and previous studies whose references are given in these papers, that permitted atomic lines are not the best indicators because of problems with the transition probabilities (although much progress has recently been made; see Biémont et al., 1991a; Hibbert et al., 1991) and of possible nonLTE effects. The best indicators are without any doubt the numerous molecular lines and particularly, the infrared lines.

We also showed (Grevesse and Sauval, 1991) how the numerous CO lines $(\Delta v=$ 1 and 2), which can now be measured with high accuracy on the ATMOS infrared solar spectra, can be used to refine the solar photospheric model of Holweger and Müller (1974) that we have used throughout these analyses.

Very recently the solar photospheric abundance of iron - which was found to be higher $\left(\mathrm{A}_{F e}=7.67\right.$ (in the usual scale where $\log N_{H}=12.00$; Blackwell et al., $1984)$ than the meteoritic value (7.51; Anders and Grevesse, 1989) - was decreased down to the meteoritic value thanks to the use of higher excitation lines of Fe I, and to lines of Fe II for which accurate gf-values have recently been obtained (Holweger et al., 1990; Holweger et al., 1991; Biémont et al., 1991b; Hannaford et al., 1992; Johansson et al., 1993).

As $\mathrm{Fe}$ is a substantial electron donor, this decreased abundance has led to modifications in the electron and gas pressures. These modifications have non-negligible effects on the temperature structure as derived from the $\mathrm{CO}$ infrared lines, and therefore on the abundances derived from the many different atomic and molecular indicators of the $\mathrm{C}, \mathrm{N}$ and $\mathrm{O}$ abundances described in Section 2 .

Work is in progress to reanalyze all these effects in detail. Preliminary results indicate that our previous results should be decreased by about $0.05 \mathrm{dex}$. Thus, the preliminary recommended values of the solar abundances of $\mathrm{C}, \mathrm{N}$ and $\mathrm{O}$ are:

$$
\begin{aligned}
& A_{C}=8.55 \\
& A_{N}=7.99 \\
& A_{O}=8.87 .
\end{aligned}
$$

The infrared $\mathrm{CO}$ vibration-rotation bands show numerous lines due to ${ }^{13} \mathrm{C}^{16} \mathrm{O}$, ${ }^{12} \mathrm{C}^{18} \mathrm{O}$, and even ${ }^{12} \mathrm{C}^{17} \mathrm{O}$ which was identified for the first time in the solar photospheric spectrum. Isotopic ratios derived from these lines agree with the terrestrial ratios but, new more accurate, transition probabilities are urgently needed for these isotopic species.

\section{Acknowledgements}

We thank W. Nijs (Brussels) for his continuous help with the calculations carried out at the Apollo computers of the Royal Observatory. We also gratefully acknowledge the hospitality and help of the ATMOS Data Facility Team (C.B. Farmer and M. Gunson) at the Jet Propulsion Laboratory. We also thank the Belgian Fonds National de la Recherche Scientifique for financial support. 


\section{References}

Anders, E., and Grevesse, N.: 1989, Geochim. Cosmochim. Acta 53, 197.

Biémont, E., Hibbert, A., Godefroid, M., Vaeck, N., and Fawcett, B.C.: 1991a, Astrophys. J. 375, 818.

Biémont, E., Baudoux, M., Kurucz, R.L., Ansbacher, W., and Prinnington, E.H.: 1991b, Astron. Astrophys. 249, 539.

Blackwell, D.E., Booth, A.J., and Petford, A.D.: 1984, Astron. Astrophys. 132, 236.

Farmer, C.B., and Norton, R.H.: 1989, A High-Resolution Atlas of the Infrared Spectrum of the Sun and the Earth Atmosphere from Space, Vol. 1, The Sun, NASA Ref. Pub. 1224, Washington, D.C.

Geller, M., Sauval, A.J., Grevesse, N., Farmer, C.B., and Norton, R.H.: 1991, Astron. Astrophys. $249,550$.

Grevesse, N., Sauval, A.J., and van Dishoeck, E.F.: 1984, Astron. Astrophys. 141, 10.

Grevesse, N., Lambert, D.L., Sauval, A.J., van Dishoeck, E.F., Farmer, C.B., and Norton, R.H.: 1990, Astron. Astrophys. 232, 225.

Grevesse, N., Lambert, D.L., Sauval, A.J., van Dishoeck, E.F., Farmer, C.B., and Norton, R.H.: 1991, Astron. Astrophys. 242, 488.

Grevesse, N., and Sauval, A.J.: 1991, in C. Jaschek and Y. Andrillat (eds.), The Infrared Spectral Region of Stars, Cambridge University Press, Cambridge, p. 215.

Hannaford, P., Lowe, R.M., Grevesse, N., and Noels, A., 1992, Astron. Astrophys. (in press).

Hibbert, A., Biémont, E., Godefroid, M., and Vaeck, N.: 1991, Astron. Astrophys. Suppl. $88,505$.

Holweger, H., and Müller, E.A.: 1974, Solar Physics 39, 19.

Holweger, H., Heise, C., and Kock, M.: 1990, Astron. Astrophys. $232,510$.

Holweger, H., Bard, A., Kock, A., and Kock, M.: 1991, Astron. Astrophys. $249,545$.

Iglesias, C., and Rogers, F.: 1991, Astrophys. J. 371, 173 and 408.

Johansson, S., Nave, G., Geller, M., Sauval, A.J., and Grevesse, N.: 1993, these proceedings.

Kurucz, R.L.: 1991, in L. Crivellari, I. Hubeny and D.G. Hummer (eds.), Stellar Atmospheres: Beyond Classical Models, Kluwer, Dordrecht.

Sauval, A.J., Grevesse, N., Brault, J.W., Stokes, G.M., and Zander, R.: 1984, Astrophys. J. 282, 330.

Seaton, M.J., Zeippen, C.J., Tully, J.A., Pradhan, A.K., Mendoza, C., Hibbert, A., and Berrington, K.A.: 1992, Revista Mexicana de Astronomia y Astrofisica 23, 19. 